\title{
Optical Design of OCT with Gapped Magnetic Ring*
}

\author{
Xiaoyi Su, Qifeng Xu \\ College of Electrical Engineering and Automation, Fuzhou University, Fuzhou, China \\ Email: xiaos_320@163.com
}

Received 2013

\begin{abstract}
The mathematics and physics model of OCT (Optical Current Transformer) with a gapped magnetic ring is briefly discussed in the paper. And some proposals of how to select the magnetic materials and crystals and reduce the stress birefringence of the crystal are also put forward in the paper. Based on the above, an OCT with $1000 \mathrm{~A}$ rated current is designed by using the ANSOFT Maxwell tools.
\end{abstract}

Keywords: Ferromagnetic Collector Type; Optics Current Transformer; Magnetic Properties; Linear Birefringence

\section{Introduction}

Faraday magneto-optical effect is applied in optical current transformer (OCT). Based on its sensing methodology it can be divided into four types: fiber one, bulk glass one, solenoid one, as well as gapped magnetic ring one [1]. The one with a gapped magnetic ring utilizes a small piece of magneto-optic crystal and other optical elements to be placed in the gap of the magnetic ring wounding around a current carrying conductor to form an optical circuit. The gap magnetically induced by the current flowing in the conductor and the crystal are the "heart" of an optical current transformer head. Taken together, the magnetic properties and the optical properties should be taken into consideration in the design. This paper discusses the characteristics of the OCT with gapped magnetic ring. Additionally, reasons and procedures to select magnetic materials and crystals and mitigate stress birefringence are also represented specifically in the paper. Depending on the analysis above and the ANSOFT Maxwell tools, the optimized structure of an OCT with 1000A has been achieved.

\section{The Characteristics of OCT with Gapped Magnetic Ring}

When a linearly polarized light passes through a magnetic field paralleling to its propagation direction, a optical phase shift of the light called Faraday rotation angle will created due to the Faraday effect

$$
\theta=V \int H d l
$$

where $V$ is the Verdet constant. Once deemed if a gap of

*Project supported by the National Natural Science Foundation of China (51177016). magnetic ring is small enough, the gap magnetic can be regarded as same as the ring inherent magnetic induced by the current flowing in the conductor. Additionally, based on the Ampere circuital theorem the gap magnetic strength is given by

$$
H_{g}=I /\left(l_{g}+u_{g} l_{a} / u_{a}\right)
$$

where $l$ is the polarized light effective optical passing path in the gap, $I$ is the primary current, $l_{g}$ is the air-gap length, $u_{g}$ is the air permeability, $u_{a}$ is the gapped magnetic ring permeability, $l_{a}$ is the gapped magnetic ring average loop length. As $u_{g}$ is far less than $u_{a}$, Equation (1) is simplified as

$$
H_{g} \approx I / l_{g}
$$

Combined Equation (1) with Equation (3), the Faraday deflection angle $\theta$ and the primary current $I$ are given by

$$
\begin{gathered}
\theta=V \int H d l=u_{g} V I l_{c} / l_{g} \\
I=l_{g} \theta /\left(u_{g} V l_{c}\right)
\end{gathered}
$$

in which $l_{c}$ is the crystal length. Set $P_{i}$ to be the polarizer output light intensity and $P_{0}$ to be the analyzer output light intensity. According to the Malus law, the relationship between $P_{i}$ and $P_{0}$ is expressed as

$$
P_{0}=a P_{i} \cos ^{2}(\varphi+\theta)
$$

where $\alpha$ is the optical attenuation coefficient intensity; $\varphi$ is the difference optical phase shift between the two output light waves.

Desiring to obtain the maximum output power, $\varphi$ is set to $45^{\circ}$. If $\theta$ is small enough, $\sin 2 \theta$ will approximately equal to $2 \theta$. Equation (6) transforms into

$$
P_{0}=\frac{a}{2} P_{i}\left(1+\frac{2 u_{g} V I l_{c}}{l_{g}}\right)=P_{D C}+P_{A C}
$$


where $P_{A C}$, the alternate current signal, is expressed as $P_{A C}=\left(a P_{i} u_{g} V I l_{c}\right) / l_{g}$, while $P_{D C}$, the direct current signal, is expressed as $P_{D C}=\left(a P_{i}\right) / l_{g}$. Eliminating the fluctuations of optical power by introducing the operation $U=P_{A C} / P_{D C}$. The relationship between $U$ and $\mathrm{I}$ is given by

$$
U=I \cdot 2 u_{g} V I l_{c} / l_{g}
$$

\section{The Optimal Design of OCT}

\subsection{The Selection of Magnetic Material}

The cold-rolled silicon steel, the permalloy alloy and the amorphous alloy are commonly made on gapped magnetic rings. Their characteristics differences are reflected in the magnetic permeability, the remanence density, the saturation magnetization, the coercive force, the iron loss, the Curie temperature, as well as the magnetostriction coefficient. Considering that the OCT actual operation environment temperature is less than the Curie temperature of magnetic materials, the iron loss and the magnetostriction coefficient are negligible under the 1000A power frequency system, so these three factors, the iron loss, the Curie temperature and the magnetostriction coefficient can be ignored. Table 1 shows the remainder typical parameters.

Table 1. The comparison of typical parameters of the three materials.

\begin{tabular}{ccccc}
\hline & \multicolumn{4}{c}{ Typical Parameters } \\
\cline { 2 - 5 } Materials & $\begin{array}{c}\text { Saturation } \\
\text { Mag- } \\
\text { netiz-ation } \\
(\mathrm{T})\end{array}$ & $\begin{array}{c}\text { Magnetic } \\
\text { permeability } \\
\left(* 10^{4}\right)\end{array}$ & $\begin{array}{c}\text { Remanence } \\
\text { density } \\
(\mathrm{T})\end{array}$ & $\begin{array}{c}\text { Co- } \\
\text { erci-ve } \\
\text { force } \\
(\mathrm{A} / \mathrm{m})\end{array}$ \\
\hline $\begin{array}{c}\text { amorphous } \\
\text { alloy }\end{array}$ & 1.5 & 0.25 & 1 & 2.0 \\
\hline $\begin{array}{c}\text { permalloy } \\
\text { alloy }\end{array}$ & 0.6 & 5.8 & 0.4 & 4.3 \\
\hline $\begin{array}{c}\text { cold-rolled } \\
\text { silicon } \\
\text { steel }\end{array}$ & 1.7 & 0.12 & 1.56 & 7 \\
\hline
\end{tabular}

Permalloy alloy has the highest initial permeability, the amorphous alloy has a lower one and the cold-rolled silicon steel has a lowest one. Additionally, for closed cores, a higher initial permeability is good for measurement sensitivity. But the gapped magnetic rings are different. As the gap magnetic induction $B_{g}$ is

$$
B_{g}=\frac{I}{\left(l_{a} / u_{a}+l_{g} / u_{g}\right)}
$$

Once the air permeability is $10^{4}$ to $10^{5}$ times bigger than magnetic rings' . Then $l_{a}$ is $10^{2}$ times larger than $l_{g}$, so $l_{a} / u_{a}$ is far less than $l_{g} / u_{g}$. Equation (9) is simplified as

$$
B_{g}=u_{g} I / l_{g}
$$

In this case, the magnetic induction $B_{g}$ depends on the primary current $I$, the gap length $l_{g}$ and the air permeability $u_{g}$. That is to say, the permeability $u_{a}$ is an unimportant factor to the magnetic properties of gapped magnetic rings.

Static field simulations on three magnetic materials shown in Table $\mathbf{1}$ are done respectively by using the ANSOFT Maxwell tool. The simulation gapped magnetic ring parameters as follow: the inner radius is $30 \mathrm{~mm}$, the outer radius is $70 \mathrm{~mm}$, the height is $40 \mathrm{~mm}$ and, the gap length is $20 \mathrm{~mm}$. Their basic magnetization curve are illustrated in Figure 1. When the primary current is less than $4 \mathrm{KA}$, these three curves are overlapped and the cold-rolled silicon steel one has the longest current linear region. Since normal load current flowing on the conductor is usually under $1 \mathrm{kA}$ level, associating with the linear region length shown in Figure 1, cold-rolled silicon steel and amorphous alloy have better magnetic performance compared to permalloy alloy.

The remanence of magnetic concentrator ring is given by[2]:

$$
B_{r}=-f(H) \cdot \frac{u_{g} l_{a}}{l_{g}}=f(H=0) \cdot \frac{u_{g} l_{a}}{l_{g}}
$$

where $f(B)$ is a closed core magnetization curve function.

The remanence in the Gapped magnetic ring are depended on the average magnetic path length and the air gap length. These dependencies make the necessity to design a right ring overall size. A smaller remanence has certain distinct advantages to maintain the response characteristics of magnetization, decrease coercive force and reduce hysteresis loss.

Taken together, the norm to select a suitable magnetic material is the one having a small remanence, a small coercive force and a large saturation magnetic flux density. Thereby, amorphous alloy is the most suitable material.

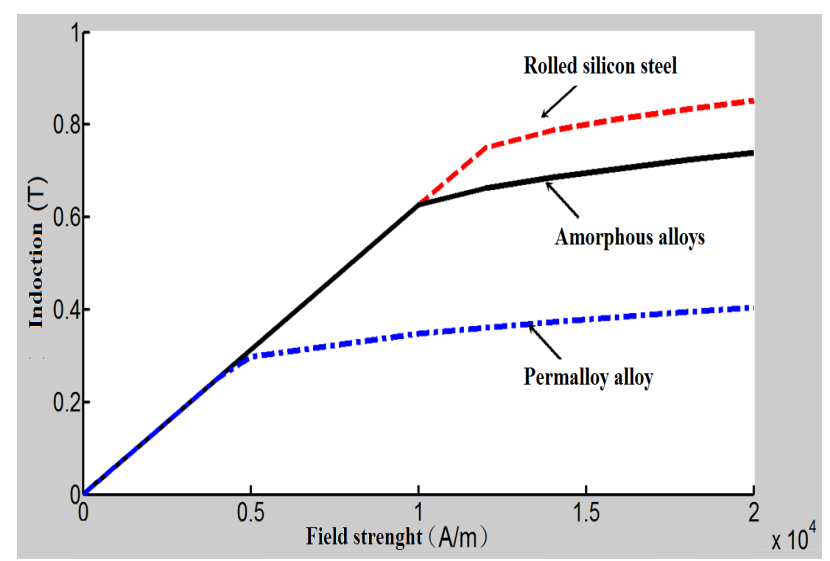

Figure 1. The basic magnetization curve of the three gap magnetic rings. 


\subsection{The Selection of Magneto-Optical Crystal}

Since the Verdet constant varies with the dynamic temperature, the temperature coefficient should be considered during the selection of magneto-optical crystal. When the temperature changes from $-25^{\circ} \mathrm{C}$ to $+80^{\circ} \mathrm{C}$, the Verdet constant of FR-5, a paramagnetic material, is reduced by $30 \%$ throughout the mutative temperature and YIG that is a ferromagnetic material has 25\% irregular variation, while $\mathrm{ZF}-7$ that is an anti-magnetic material only changes $0.79 \%$ [3]. Similarly, an anti-magnetic material called MR1 almost has no effect on the Verdet constant in the temperature ranging from -55 to $+135^{\circ} \mathrm{C}$. Additionally, its Verdet constant is as high as 0.065 0.092 min/Oe.cm (@632.8 nm) which is helpful to shorten the crystal length and reduce the line birefringence. Therefore, the MR1 is employed in this design.

As the OCT using light to measure the magnetic field surrounding a current carrying conductor has a transfer function with a sine wave characteristics which made the OCT polarization interference be a non-linear portion. An approximate linear relationship between $\sin 2 \theta$ and $2 \theta$ introduced in the section 2 can fix this problem when $\theta$ is small enough. Ultimately, the OCT accuracy level will determine the maximum Faraday deflection angle and the crystal length. Set $X$ is the difference between $l_{g}$ and $l_{c}$, Equations (5) could be transformed as

$$
l_{c}=\frac{\theta X}{u_{g} V I-\theta}
$$

Assuming the OCT accuracy is a 0.2 class and the primary current is $1000 \mathrm{~A}$, its measurement error is less than $0.2 \%$. Then the maximum Faraday deflection angle $\theta_{\max }$ is $0.877^{\circ}$. Set the maximum $X$ is $20 \mathrm{~mm}$, (As the prism and polarizer product class size are $7 \mathrm{~mm}$ and 2 $\mathrm{mm}$ respectively. The other $2 \mathrm{~mm}$ space is reserved for installation). Putting these data into Equation (12), $l$ is calculated to $16.72 \mathrm{~mm}$. Therefore, the crystal maximum length is $16 \mathrm{~mm}$.

\subsection{Methods to Reduce Stress Birefringence}

During the process of magneto-optic crystal production and processing or the interaction between the sensor head and the adjacent structure, residual stress will occur and stress birefringence will created simultaneously.

Since expansion coefficient has a function with the temperature when the temperature changes the difference on the materials expansion coefficient will cause temperature gradients owing to the uneven temperature distribution of the sensing head. Then thermal stress is generated within the sensing head and a linear birefringence appears. Line birefringence aliasing on the Faraday polarization angle can undermine the reliability and stability of the OCT [4]. For example, an OCT sensing head has a structure of $70 \mathrm{~mm}$ outer diameter, $30 \mathrm{~mm}$ inner diameter, $40 \mathrm{~mm}$ height and $30 \mathrm{~mm}$ air gap length and ZF-7 as the sensing element which is $10 \mathrm{~mm}$ long, conducted by a 1000 A primary current, its Faraday rotation angle is $4.81^{\circ}$ and its line birefringence angle is about $0.56^{\circ}$ and it introduces $11.64 \%$ measurement error. The method to reduce the linear birefringence is to reduce the magneto-optical material length. For example, using a 200 um thick magneto-optical film that dopes with $\mathrm{Ce}^{3+}$ by using the technology of liquid phase epitaxial (LPE) as the sensing element instead of ZF-7 [5], then the Faraday deflection angle is turned to $28.6^{\circ}$ and its line birefringence angle is $0.0112^{\circ}$ only and the corresponding error drops to $0.039 \%$. It turns that the influence of linear birefringence on Faraday deflection angle is effectively weakened.

Further, filling asbestos in sensor head or utilizing materials having the similar expansion coefficient with the magneto-optical material can also free from the temperature variation gradient and weaken the thermal stress birefringence.

\subsection{The Optimization Structure of Gapped Magnetic Rings}

Since the gapped magnetic ring's inner radius $r$ is determined by the size of a current carrying conductor which is determined by the primary current. Depending on the analysis, the rated current of an OCT designed in this paper is $1000 \mathrm{~A}$, so conductive rod radius is set to $18 \mathrm{~mm}$. Additionally, leaving a $10 \mathrm{~mm}$ margin space to fill asbestos, the gapped magnetic ring inner radius $\mathrm{r}$ is $28 \mathrm{~mm}$. Assuming $N$ is the difference between the inner and outer radius of the gapped magnetic ring. So the outer radius $R$ can be expressed as the sum of $r$ and $N$. Combing Equation (3) with Equation (11), the gap length not only determines the gap induction, but also affects the remanent density. when the magnetic material is certain, the smaller the gap length, the greater the gap induction and remanence. Since the crystal length is described in selection 3.2, the air gap has a length ranging from $20 \mathrm{~mm}$ to $36 \mathrm{~mm}$. Depending on the ANSOFT Maxwell tool, making simulations on three gapped magnetic rings with 25 $\mathrm{mm}, 30 \mathrm{~mm}$ and $35 \mathrm{~mm}$ penning gap length under the 1000 A system respectively. The result are illustrated in Figure 2 and Figure 3 which show the effect of the difference between a gapped magnetic ring inner and outer radius on the gap induction $B_{g}$, as well as on the remanent $B_{r}$.

The larger the difference between a gapped magnetic ring's inner and outer radius $N$, the more uniform gap magnetic field distribution and the smaller the magnetic flux leakage [6]. When $N$ exceeds the length $30 \mathrm{~mm}$, the gap magnetic distribution is completely uniform. Considering to decrease the volume of material and achieve 


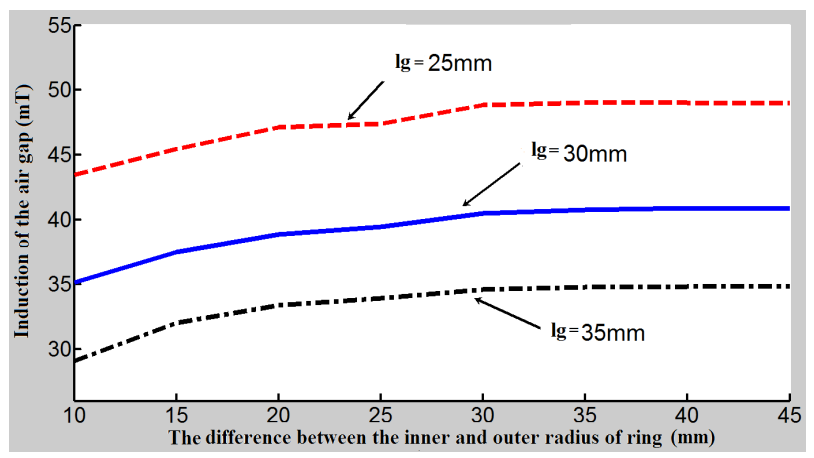

Figure 2. The effect of the difference between a gapped magnetic ring's inner and outer radius on the gap induction.

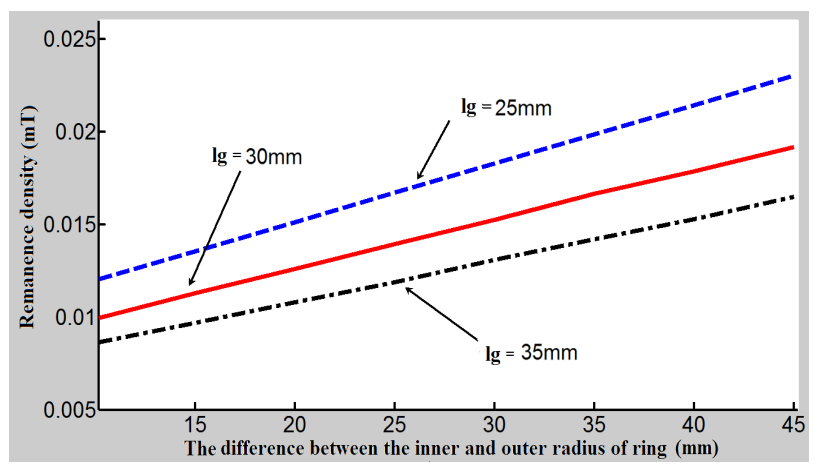

Figure 3. The effect of the difference between a gapped magnetic ring's inner and outer radius on the remanent.

the optimal performance of the OCT, $N$ is set to $30 \mathrm{~mm}$, thereby, the gapped magnetic ring outer radius $R$ is 58 $\mathrm{mm}$. Additionally when $N$ is certain, the longer the gap length, the smaller the remanence density. As the three remanence density curves are at $10^{-2} \mathrm{mT}$ level in Figure 3. Comparing to the amorphous alloy saturation magnetic flux density $1.5 \mathrm{~T}$, the remanence density of ring made of amorphous alloy can be ignored. Therefore, under the 1000 A system, the analysis of the OCT transient characteristics can ignore the effects of remanence. Fortunately, a smaller gap length is helpful to obtain a large induced magnetic field, the length of air gap is set to 25 $\mathrm{mm}$, correspondingly the magneto-optical crystal length is set to $5 \mathrm{~mm}$.

\section{Conclusions}

This paper discusses the characteristics of OCT with gapped magnetic ring, and some considerations to select the magnetic material and the magneto-optical crystal, and reduce the stress birefringence. Depending on the analysis in the paper, the optimized design of an OCT with 1000 A rated primary current is designed by using the ANSOFT Maxwell tools. This optimized sensing head has a structure as follows: $28 \mathrm{~mm}$ inner radius, 58 $\mathrm{mm}$ outer radius, $15 \mathrm{~mm}$ height and $25 \mathrm{~mm}$ length gap. Additionally, the magneto-optical crystal choose MR1 with the length of $5 \mathrm{~mm}$. In a temperature range from -40 ${ }^{\circ} \mathrm{C}$ to $+80^{\circ} \mathrm{C}$, the OCT designed can be employed successfully.

\section{REFERENCES}

[1] Y. B. Liu, H. B. Li, C. Y. Yu, G. X. Ye and X. Q. Wang, "The Principle, Technology and Application of Electronic Transformer,” Science Press, Beijing, 2009, pp. 11-35.

[2] B. B. Afanasyev, "Current Transformer," Mechanical Industry Press, Beijing, 1989, pp. 156-160.

[3] Z. P. Wang, X. Z. Wang and Q. B. Li, "Theoretical Analysis of Temperature Characteristics of a Bulk Glass Optical Current Sensing Element with Polarization-Preserving Layers," Acta Photonic Sinica, Vol. 35, No. 6, 2006, pp. 846-849.

[4] Z. P. Wang, Q. Wu, Q. B. Li, Z. J. Huang and J. H. Shi, "Theoretical Analysis of the Effect of the Temperature Features of Linear Birefringence on the Performance of an Optic-glass Current Sensor," Journal of Harbin Engineering University, Vol. 26, No. 2, 2005, pp. 272-276.

[5] M. Huang and S. Y. Zhang, "Crystal Growth and Magneto-optical Properties of Ce3+ Doped Rare-earth Iron Garnet," Chinese Journal of Materials Research, Vol. 14, No. 4, 2000, pp. 393-396.

[6] Y. Yoshida, S. Kawazoe, K. Ibuki, K. Yamada and N. Ochi, "New Fault Locating System for Air-insulated Substation Using Optical Current Detector," IEEE Transactions on Power Delivery, Vol. 7, No. 4, 1992, pp. 1804-1813.

doi: $10.1109 / 61.156982$ 